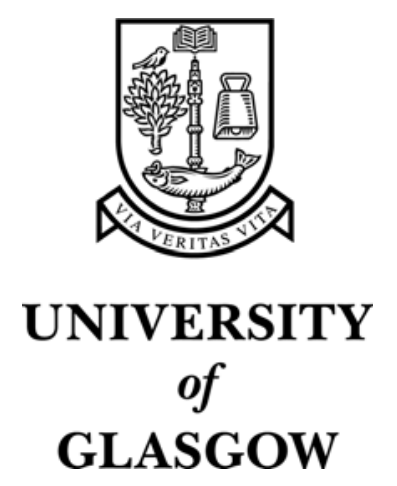

Wall, S.A. and Brewster, S.A. (2003) Assessing haptic properties for data representation. In, SIGCHI Conference on Human Factors in Computing Systems, 5-10 April 2003, pages pp. 858-859, Ft. Lauderdale, Florida.

http://eprints.gla.ac.uk/3276/ 


\title{
Assessing Haptic Properties for Data Representation
}

\author{
Steven A. Wall and Stephen A. Brewster \\ Glasgow Interactive Systems Group, Department of Computing Science \\ University of Glasgow, Glasgow, G12 8QQ, UK. \\ Tel: +44 (0)141330 4966 \\ Email: steven, stephen@dcs.gla.ac.uk Web: http://www.dcs.gla.ac.uk/ steven
}

\begin{abstract}
This paper describes the results of a series of forced choice design experiments investigating the discrimination of material properties using a PHANToM haptic device. Research has shown that the PHANToM is effective at displaying graphical information to blind people, but the techniques used so far have been very simple. Our experiments showed that subjects' discrimination of friction was significantly better than that of stiffness or the spatial period of sinusoidal textures, over the range of stimuli investigated. Thus, it is proposed that graphical data could be made more easily accessible to blind users by scaling the data values to friction rather than shape or size, as in traditional bar charts.
\end{abstract}

\section{Keywords}

Haptic, PHANToM, material properties, discrimination, blind, visually impaired.

\section{INTRODUCTION}

Visualisations such as line graphs and bar charts are frequently used to illustrate trends and distributions of data in a simple and intuitive way. They are commonly used in subjects such as economics, mathematics and the sciences. Being unable to access graphical information is a common obstacle to blind people who wish to pursue scientific based studies or careers. Traditional methods to overcome this include presenting graphs as raised lines on specially prepared paper. The commercial availability of haptic devices, such as the PHANToM, has presented the opportunity to render data stored on a computer as haptic graphs for blind users. This provides a richer and more flexible way for blind students to access the information.

Current implementations of haptic graphs for blind and visually impaired computer users have adopted a direct analogy with their visual counterparts. A haptic device is used to explore the height of bars, shape of lines or the contours of a surface plot. However, visualisations such as these rely on the distributed nature of the visual sense to identify trends in the data through perception and

COPYRIGHT IS HELD BY THE AUTHOR/OWNER(S).

CHI 2003, APRIL 5-10, 2003, FT. LAUDERDALE, FLORIDA, USA. ACM 1-58113-630-7/03/0004. comparison of shape and size. Conversely, the touch sense is very localised. The "point interaction" nature of devices such as the PHANToM further exasperates the problem by limiting the cutaneous feedback available to the user. The rich, spatially distributed nature of visual cues is not available; hence, users must successfully integrate "temporally varying" cues as they traverse the objects or surfaces. For large or complex data, this places considerable short-term memory demands on the user, thus reducing performance, and comprehension of the data.

A more successful approach may be to display the data by scaling properties that are more quickly and easily perceived by the haptic sense. Klatzky, Lederman and Reed [1] showed that during a sorting task with real objects, subjects discriminated stimuli visually using structural properties (size and shape cues), whereas when working haptically they relied more on material cues such as compliance and texture. Thus, in an application to display bar charts to blind users, bars with a low value could be very compliant (low stiffness), whilst those with a high data value could be rigid to touch (high stiffness). The purpose of the experiment presented here was to investigate the ability of participants to discriminate three common material properties displayed using a PHANToM haptic device. These were friction, stiffness and the spatial period of a sinusoidal texture. The ability to discriminate properties such as these has been well documented for physical objects, but has not been investigated using virtual stimuli presented with a PHANToM device.

\section{EXPERIMENTAL STIMULI}

The stimuli for the experiment were rendered using the GHOST software development kit from Sensable Technologies Inc. For the stiffness condition, the surface spring stiffness of the object was varied. For the friction condition, both the static and dynamic friction were varied equally. For the texture condition, a GHOST force field effect was created that rendered lateral sinusoidal forces when the PHANToM was in contact with the surface. It has previously been shown in other studies that purely lateral forces can create an illusion of texture or bumps [4]. The parameter varied for the texture condition was the spatial period. The "roughness" of the sinusoidal textures was intentionally not referred to, as previous studies have highlighted difficulties in establishing relationships between spatial period and roughness [3]. 


\section{EXPERIMENTAL PROCEDURE}

Twelve sighted subjects participated in the experiment. They were all recruited from the student population of the Department of Computing Science, and were all paid for their participation. The experiment adopted a withinsubjects forced choice methodology, whereby participants chose the "odd one out" from three surfaces that varied in one of the three parameters for each condition. During each step of the experiment, subjects were presented with the three surfaces that were represented using the PHANToM. The following values were assigned to the three properties as "standard" values: stiffness $500 \mathrm{~N} / \mathrm{m}$, friction $0.5 \mathrm{Ns} / \mathrm{m}$, sinusoid spatial period $3 \mathrm{~mm}$. Prior to each experimental condition, the subject was instructed that the stiffness, friction or texture would be varied. The parameters that were not varied in a condition were held constant at the standard values (except for texture, which was not present in the stiffness and friction conditions). The subject was also instructed how best to explore the surfaces in order to perceive the relevant quality. These were based on the exploratory procedures (EP) of Lederman and Klatzky [2]. For stiffness, the subjects were instructed to tap on the surface. For both friction and texture, the subjects were instructed to move the stylus laterally, up and down the surface.

For each step in a condition one of the three surfaces, chosen at random, was designated as the "test surface". The test surface varied in the relevant parameter (friction, stiffness or spatial period) by $\pm 10 \%, \pm 20 \%, \pm 30 \%, \pm 40 \%$ or $\pm 50 \%$ from the standard value. Each difference was presented nine times, resulting in 90 steps per condition. The task for each step was to select which of the surfaces was the test surface. Each subject took part in three conditions, one for each surface property. The order of conditions was counterbalanced between the subjects. For all conditions the subject wore headphones playing filtered white noise in order to mask the audible cues produced by the PHANToM during operation.

\section{RESULTS AND DISCUSSION}

Figure 1 shows the proportion of correct responses averaged across all subjects for the three conditions. A two-way repeated measures ANOVA was performed on the data, with the independent variables being stimuli (stiffness, friction or texture) and percentage difference between test and standard surface. The dependant variable was the proportion of correct responses given by the participants. It was found that the type of stimuli had a significant effect on performance $(\mathrm{F}(2,165)=14.61$, $\mathrm{P}<0.001)$, as did the percentage difference from the standard $(\mathrm{F}(4,165)=74.48, \quad \mathrm{P}<0.001)$. There was no significant interaction between the two variables $(\mathrm{F}(8,165)=0.473, \mathrm{P}>0.05)$. Post hoc Tukey tests revealed that the performance with friction was significantly better than with texture $(\mathrm{P}<0.001)$ or stiffness $(\mathrm{P}<0.001)$, however, there was no significant difference between texture and stiffness $(\mathrm{P}>0.05)$. Thus, participants were better able to resolve stimulus changes in friction than in either of the other two parameters.

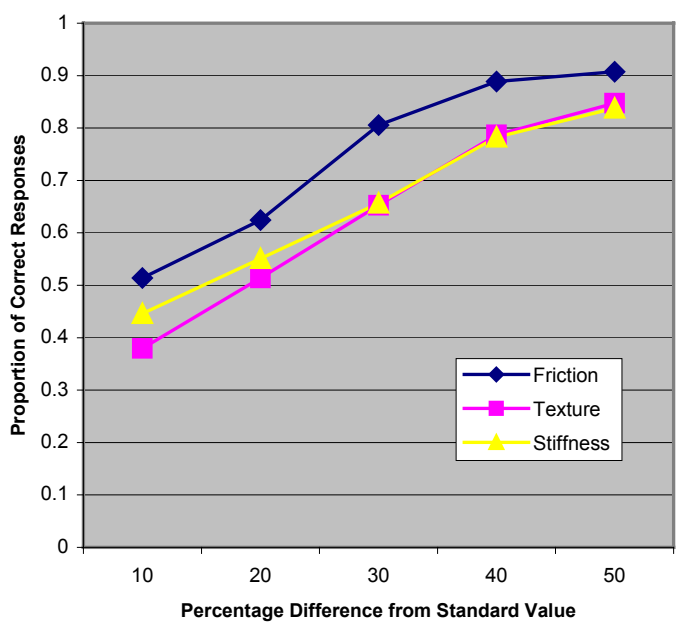

Figure 1: Proportion of Correct Responses for Percentage Difference from Standard Value

In addition, it was also noted that discrimination was significantly better for negative changes in stimuli relative to the standard value (lower stiffness, friction and spatial period) compared to positive changes. This suggests a nonlinear relationship between the values of the haptic properties and the perceived magnitude. Further experiments will seek to identify these relationships, such that "standard" surfaces can be chosen for optimum sensitivity to variations.

In conclusion, for the chosen standard surfaces in this experiment, subjects were able to discriminate changes in friction significantly better than for both stiffness and spatial period of texture. It is therefore proposed that the level of friction of bars in a haptic bar chart application could be scaled to the data values, thus potentially increasing speed and efficiency for blind users over standard visually-based representations.

\section{REFERENCES}

1. Klatzky, R.L., Lederman, S.J. and Reed, C. There's More to Touch than Meets the Eye: The Salience of Object Attributes for Haptics with and without Vision, Journal of Experimental Psychology 116, 4 (1987), 356-369.

2. Lederman, S.J. and Klatzky, R.L. Hand Movements: A Window into Haptic Object Recognition, Cognitive Psychology 19, 3 (1987) 342-368.

3. McGee, M.R., Gray, P. and Brewster, S.A. Feeling Rough: Multimodal Perception of Virtual Roughness, In: Eurohaptics 2001: Conference Proceedings, University of Birmingham, July 2001, 29-33.

4. Minsky, M., Ouh-Young, M., Steele, O., Brooks, F.P. and Behensky M. Feeling and Seeing: Issues in Force Display, Computer Graphics, Vol. 24, No. 2 (March 1990), 235-243. 